The Interplay of Oxytocin and Collectivistic Orientation Shields against Negative Effects of Ostracism

\author{
Michaela Pfundmair ${ }^{1}$ \\ Nilüfer Aydin ${ }^{1}$ \\ Dieter Frey $^{1}$ \\ Gerald Echterhoff ${ }^{2}$ \\ ${ }^{1}$ Ludwig-Maximilians University of Munich \\ Leopoldstr. 13, 80802 München, Germany \\ ${ }^{2}$ Westfälische Wilhelms-University Münster \\ Fliednerstr. 21, 48149 Münster, Germany \\ g.echterhoff@uni-muenster.de
}

michaela.pfundmair@psy.lmu.de, aydin@psy.lmu.de, dieter.frey@psy.lmu.de

Published in: Journal of Experimental Social Psychology 55 (2014) 246-251

http://dx.doi.org/10.1016/j.jesp.2014.07.016

Contact Details:

Michaela Pfundmair

LMU München

Leopoldstr. 13

80802 Munich, Germany

Tel. +49 8921803002

Email: michaela.pfundmair@psy.lmu.de 


\begin{abstract}
Surprisingly, oxytocin, a socially potent neuropeptide, has not been found to affect responses to ostracism. However, these effects may depend on individual differences, specifically the propensity for cooperation and social connectedness. We thus predicted that oxytocin is more likely to attenuate negative responses to ostracism in people with collectivistic (vs. individualistic) orientation, particularly horizontal collectivism, which is oriented toward cooperation among equals. After intranasal administration of oxytocin or a placebo, participants were ostracized or included during the computer-based ball-tossing game Cyberball and indicated their sense of social comfort regarding the Cyberball game. Under oxytocin, responses to ostracism were less negative in participants with a horizontal collectivistic (vs. individualistic) orientation. The results could not be explained by affect, including anxiety. The results suggest that collectivistic beliefs facilitate oxytocin's shielding effect against negative consequences of ostracism. We discuss underlying mechanisms and factors other than collectivistic orientation that could explain the findings. (149 words)
\end{abstract}

Keywords: ostracism; oxytocin; collectivistic versus individualistic orientation, social connectedness; need for belonging 


\section{The Interplay of Oxytocin and Collectivistic Orientation Shields against Negative}

\section{Effects of Ostracism}

Being socially excluded or even ostracized triggers a plethora of negative responses in humans. Ostracism activates, inter alia, neuronal responses similar to physical pain (Eisenberger, Lieberman, \& Williams, 2003) and reduces the sense of belonging and selfesteem (Zadro, Williams, \& Richardson, 2004). Such consequences are hard, or perhaps impossible, to eliminate, but they can be attenuated by, for instance, long-term administration of pain suppressant (DeWall et al., 2010) or mental simulation of physical invulnerability (Huang, Ackerman, \& Bargh, 2013). Regarding neuroendocrinological factors that could mitigate negative consequences of ostracism, a prime candidate is oxytocin - a neuropeptide with potentially powerful prosocial effects (e.g., Kosfeld, Heinrichs, Zak, Fischbacher, \& Fehr, 2005).

Oxytocin has been found to inhibit discomfort and withdrawal in the face of adverse or anxiety-provoking social situations (Evans, Shergill, \& Averbeck, 2010; Radke, Roelofs, \& de Bruijn, 2013; for reviews, see De Dreu, 2012a; Kemp \& Guastella, 2011). The reduction of discomfort and withdrawal tendencies is a mechanism that can allow oxytocin to temper negative responses to ostracism.

Surprisingly, oxytocin effects on people's responses to ostracism have not yet been documented. In the only published study (Alvares, Hickie, \& Guastella, 2010), participants were ostracized (vs. included) in the computer-based ball-tossing game Cyberball (Williams, Cheung, \& Choi, 2000) after intranasal administration of oxytocin (vs. a placebo). However, there was no evidence that oxytocin attenuated the ostracism-induced decline in the sense of social comfort. Thus, quite surprisingly, oxytocin was not found to affect responses to ostracism. (We note that a study with rodents found protective oxytocin effects against longterm isolation; Grippo, Trahanas, Zimmerman, Porges, \& Carter, 2009.) 
Drawing on research demonstrating the context-sensitivity of oxytocin effects (Bartz, Zaki, Bolger, \& Ochsner, 2011; Kemp \& Guastella, 2011), Alvares and colleagues (2010) suggested that a lack of positive social cues might have prevented oxytocin effects on responses to ostracism. However, the social context is not the only potential moderator of oxytocin effects. A growing body of research suggests that oxytocin effects on social behavior can also be moderated by interindividual differences, specifically, individual factors related to people's relationship experiences and socio-emotional functioning (Radke et al,, 2013; for reviews, see Bakermans-Kranenburg \& Van IJzendoorn, 2013; Olff et al., 2013).

We focus here on a key individual characteristic that could facilitate oxytocin effects on responses to ostracism — a person's collectivistic (vs. individualistic) worldview (Triandis, 1995). Individualism and collectivism have been initially shown to vary across cultures (Triandis \& Gelfand, 2012), but they can also vary within cultures (Oyserman, Coon, \& Kemmelmeier, 2002). Collectivism encompasses a greater attunement to the concerns of one's group (vs. one's personal concerns), a sense of connectedness with others (Oyserman \& Lee, 2008), and an interdependent self-construal (Markus \& Kitayama, 1991). Due to their socio-centric commitment, people in collectivistic (vs. individualistic) cultures are driven less by personal concerns and tend to stay even in unpleasant relationships (U. Kim, 1994). There is initial evidence for the role of collectivism in oxytocin effects: In a study by Kim et al. (2011), the same oxytocin receptor gene was associated with either increased or decreased suppression of emotions in social situations depending on whether the participants lived in a collectivistic or individualistic culture.

Presumably, collectivist (vs. individualist) participants can more easily activate knowledge of positive social relations. Because their sense of connectedness is anchored in a web of social relations, collectivists should be better protected against a single ostracism experience. Indeed, recent studies suggest that people with a collectivistic outlook recover more easily from ostracism (Pfundmair, Graupmann, Frey, \& Aydin, 2014; Ren, Wesselmann, 
\& Williams, 2013). Accordingly, people with a collectivistic orientation might be better equipped for maintaining a sense of social comfort in the face of ostracism (see Knowles, 2013).

This psychological equipment could facilitate effects of exogenous oxytocin on responses to ostracism. Specifically, we argue that the threshold for protective, discomfortreducing effects of oxytocin is lower, and thus more likely to be reached, in people who have a greater (vs. lower) collectivistic orientation. When people already feel safely anchored in their interpersonal net to a greater extent (as is true for collectivists), they can be more easily "comforted" by oxytocin after adverse social experiences. This conceptualization is consistent with research showing oxytocin reduces avoidance of discomforting social stimuli (angry faces) to a greater extent in participants who report lower social anxiety and feel safer in social situations (Radke et al., 2013). According to our rationale, then, oxytocin should be more likely to prevent negative responses in people who are already protected by a collectivistic orientation.

We also suspect that some facets of collectivism are more likely to be effective in this respect. Whereas the vertical type of collectivism emphasizes social obligations from hierarchies and traditional roles, implying an acceptance of inequality, the horizontal type of collectivism involves a propensity for cooperative interaction among equals (Singelis, Triandis, Bhawuk, \& Gelfand, 1995; Triandis \& Gelfand, 1998). While both types are characterized by socio-centrism, vertical collectivism capitalizes on concerns about fulfilling one's duties within a social structure. Horizontal collectivism, in turn, capitalizes on the need for interpersonal connectedness (Triandis \& Gelfand, 2012). Thus, we propose that a collectivistic orientation, especially of the horizontal type, is an appropriate personal condition that facilitates protective effects of oxytocin. Accordingly, oxytocin should be more likely to alleviate negative responses to an experimentally induced ostracism experience in people with a horizontal collectivistic (vs. individualistic) orientation. 
To test this prediction, participants intranasally administered either oxytocin or a placebo and were then ostracized or included during Cyberball (Williams et al., 2000), a wellestablished procedure to induce strong feelings of being excluded ${ }^{1}$ (e.g., Wesselmann, Wirth, Pryor, Reeder, \& Williams, 2013), even when participants are obviously ostracized merely by a computer (Zadro et al., 2004). We then assessed participants' feeling of social comfort, as indicated by the sense of belonging and socially reflected self-esteem. We chose these two scales, which have been frequently employed in ostracism research, because they are related to affiliation behavior (Bernstein, Sacco, Brown, Young, \& Claypool, 2010; Williams, 2009) and are apt to capture the expected social effects of oxytocin (Bartz et al., 2011). We predicted that under oxytocin (vs. placebo), the drop in social comfort triggered by ostracism (vs. inclusion) should be weaker in participants with a horizontally collectivistic (vs. individualistic) orientation. Thus, responses to ostracism should be less negative in collectivistic participants after oxytocin (vs. placebo) administration, whereas this alleviation should be lower or absent in individualistic participants.

\section{Method}

\section{Participants}

Seventy-eight male students from a German university (mean age=24.97 years, $S D=7.38$ ) participated in a study ostensibly investigating hormonal effects on mental visualization. Negative selection criteria were self-reported symptoms of depression, bipolar, panic, psychotic disorders, substance dependence, epilepsy, allergies, and hypersensitivity to preservatives in the oxytocin spray. Two participants who ignored instructions were excluded, resulting in the above sample. Participants were told to abstain from alcohol and caffeine 24 hours before, and from food and drink (except water) 2 hours before arrival. Written informed consent was obtained. The study was approved by the LMU Munich Ethics Committee.

\section{Design and procedure}


The experiment was based on a 2 (Cyberball Experience: ostracism, inclusion) X 2 (Substance: oxytocin, placebo) design with random assignment to conditions. Social Orientation (from individualistic to collectivistic) served as a continuous moderator variable.

First, participants self-administered either 24 I.U. (three puffs per nostril) of oxytocin ( $n=38$; Syntocinon Spray, Novartis) or a placebo ( $n=40$; sodium chloride solution) under experimenter supervision. They then completed a social-orientation (IND-COL) questionnaire $^{2}$. After a 40-min period, which allows oxytocin to be effective (Born et al., 2002), Cyberball was started. Subsequently, responses to Cyberball (social comfort) and affective responses were assessed. Finally, participants were debriefed, and paid (€7).

\section{Materials}

Ostracism (Cyberball experience). We employed Cyberball (Williams \& Jarvis, 2006) to manipulate ostracism. Participants were led to believe that Cyberball concerns mental visualization skills, and that they were playing with two other participants on an Internet platform. However, unbeknownst to participants, the other players were computercontrolled to show the following behavior: During 40 throws, the participants received the ball roughly one third of the time (inclusion) or twice at the beginning and then no more again (ostracism).

Social orientation. Participants completed a German version (Graupmann, Jonas, Meier, Hawelka, \& Aichhorn, 2012) of the Individualism and Collectivism (IND-COL) scale (Singelis et al., 1995) on 9-point Likert scales from 1 (not at all) to 9 (very much). The mean score for 8 horizontal individualism items (Cronbach's $\alpha=.76$ ) was subtracted from the mean score for 8 horizontal collectivism items (Cronbach's $\alpha=.76$ ). The mean score for 8 vertical individualism items (Cronbach's $\alpha=.81$ ) was subtracted from the mean score for 8 vertical collectivism items (Cronbach's $\alpha=.67$ ). Higher values of the aggregate difference scores reflect a more collectivistic orientation. 
Manipulation check. The effect of the Cyberball manipulation was assessed by two items ("What percent of the throws were thrown to you?", "To what extent were you included by the other participants during the game?"; the latter on a 9-point scale; Zadro et al., 2004).

Responses to Cyberball. To assess participants' sense of social comfort in response to ostracism (vs. inclusion), we asked them to indicate their sense of belonging and sociallyreflected self-esteem (Zadro et al., 2004) ${ }^{3}$ on 9-point Likert scales from 1 (not at all) to 9 (very much), translated into German: "I felt poorly accepted by the other participants", "I felt as though I had made a 'connection' or bonded with one or more of the participants during the Cyberball game", "I felt like an outsider during the Cyberball game", "During the Cyberball game, I felt good about myself", "I felt that the other participants failed to perceive me as a worthy and likeable person", "I felt somewhat inadequate during the Cyberball game" (Cronbach's $\alpha=.82)$.

Affective Responses. After the Cyberball game, participants indicated their affect on the Positive and Negative Affect Schedule (PANAS; Watson, Clark, \& Tellegen, 1988) on Likert scales from 1 (not at all) to 5 (very much). We calculated an aggregate score for the 10 positive (Cronbach's $\alpha=.86)$ and 10 negative items ( $\alpha=.84$ ), with higher values indicating more positive affect. To control for possible effects of anxiety, we created a mean score for the items "distressed", "scared", and "nervous" $(\alpha=.72)$.

\section{Results}

We report two-tailed tests, and Cohen's $d$ or $f^{2}$ as effect sizes.

Manipulation Check. As expected, participants in the ostracism condition reported receiving significantly less throws $(M=6.84, S D=6.21)$ than did participants in the inclusion condition $(M=38.28, S D=14.12), t(76)=-12.61, p<.001, d=-2.89$. Ostracized participants felt significantly more excluded $(M=7.66, S D=1.92)$ than did included participants $(M=3.23$, $S D=1.95), t(76)=10.10, p<.001, d=2.32$. There was no evidence that participants in the oxytocin and placebo groups differed in their social orientation, $t<1, n s$. 
Interplay of oxytocin and social orientation (horizontal IND-COL). To test the hypothesized facilitation of oxytocin effects by a horizontal collectivistic orientation, we calculated a multiple regression with Cyberball experience (ostracism vs. inclusion, centered), substance (oxytocin vs. placebo, centered), social orientation (individualistic vs. collectivistic, centered by standardization), and all interaction terms as predictors. Figure 1 shows the mean scores, for social orientation plotted at $1 S D$ above and $1 S D$ below $M$ (Aiken \& West, 1991). In the following, we focus on significant regression results. A complete overview of the regression findings is given in Table 1.

Participants responded to ostracism with a lower sense of social comfort, as indicated by a significant main effect of Cyberball experience, $b=-1.170, S E=.15, t(70)=-8.00, p<.001$, $f^{2}=.821$ (for descriptive statistics, see Table 2). As can be seen in Figure 1, a drop of social comfort after ostracism (vs. inclusion) was found regardless of social orientation and substance: All four simple slopes were significantly negative, ranging from $b=-1.559$ to $b=$ $-.593, p s<.048$

Importantly, the regression analysis also revealed a significant three-way Cyberball Experience X Substance X Social Orientation interaction, $b=.388, S E=.15, t(70)=2.57$, $p=.012, f^{2}=.048$. Slope difference tests (Dawson \& Richter, 2006) showed that the drop of social comfort under ostracism (vs. inclusion) was less steep (i.e., reduced) under oxytocin, $b=-.593$, than under placebo, $b=-1.557$, in collectivistic participants, $t(70)=2.30, p=.025$. No such effect was found for individualistic participants, $t(70)=-1.40, p=.166$. Also, the effect of ostracism was less steep (i.e., reduced) in collectivistic participants than in individualistic participants, $b=-1.559$, under oxytocin, $t(70)=2.15, p=.035$. No such pattern emerged under placebo, $t(70)=-1.48, p=.144$.

To further probe the three-way interaction, we conducted separate analyses for the oxytocin and placebo group (Figure 1, top and bottom panel, respectively). The Cyberball Experience X Social Orientation interaction was not significant in the placebo group, $b=-.292$, 
$S E=.23, t(36)=-1.25, p=.219, f^{2}=.025$, but it was significant in the oxytocin group, $b=.483$, $S E=.18, t(34)=2.68, p=.011, f^{2}=.093$. Simple slope analyses revealed that under oxytocin ostracized collectivistic (vs. individualistic) participants exhibited more social comfort, $b=.807, S E=.27, t(34)=2.96, p=.006$, whereas no such effect was found for included collectivistic (vs. individualistic) participants, $b=-.160, S E=.24, t(34)=-0.68, p=.503$. Facing ostracism, oxytocin-administering collectivistic participants retained a higher sense of social comfort than did oxytocin-administering individualistic participants. In sum, social orientation moderated the impact of oxytocin on responses to ostracism (vs. inclusion) in the predicted ways.

Interplay of oxytocin and social orientation (vertical IND-COL). In our sample, the correlation between horizontal and vertical collectivism was $r(78)=.504, p<.001$. To explore the specificity of the present collectivism effects, we ran the main analyses with vertical instead of horizontal IND-COL scores. Again, we found a significant main effect of Cyberball experience, $b=-1.136, S E=.15, t(70)=-7.44, p<.001, f^{2}=.724$. There was, however, no significant Cyberball Experience X Substance X Social Orientation interaction, $b=.225$, $S E=.16, t(70)=1.45, p=.150, f^{2}=.016$. Thus, there was no evidence that vertical collectivism had the same effect as horizontal collectivism.

\section{Interplay of oxytocin and integrated social orientation scores (vertical and}

horizontal IND-COL). However, the main findings remained largely unchanged when we used total IND-COL scores, including both the vertical and horizontal type (for details, see Supplemental Materials). We again found a significant main effect of ostracism, $b=-1.152$, $S E=.15, t(70)=-7.82, p<.001, f^{2}=.689$, and, importantly, a significant Cyberball Experience $\mathrm{X}$ Substance X Social Orientation interaction, $b=.335, S E=.15, t(70)=2.22, p=.030, f^{2}=.037$. A slope difference test revealed that the drop of social comfort under ostracism (vs. inclusion) was less steep (i.e., reduced) under oxytocin (vs. placebo) in more collectivistic participants, $t(70)=1.95, p=.055$; this pattern was not found for individualistic participants, $t(70)=-1.23$, 
$p=.223$. Also, the effect of ostracism tended to be less steep in collectivistic (vs.

individualistic) participants under oxytocin, $t(70)=1.81, p=.074$. No such pattern emerged under placebo, $t(70)=-1.31, p=.194$.

Separate regression analyses for the oxytocin and placebo group revealed a significant Cyberball Experience X Social Orientation interaction in the oxytocin group, $b=.424, S E=.19$, $t(34)=2.23, p=.032, f^{2}=.070$. No such interaction effect was found in the placebo group, $b=-$ $.247, S E=.22, t(36)=-1.10, p=.277, f^{2}=.019$. Under oxytocin individualistic and collectivistic participants appeared to differ in the ostracism condition, as suggested by a simple slope test, $b=.563, S E=.28, t(34)=2.01, p=.052$. This was not the case in the inclusion condition, $b=-.284$, $S E=.26, t(34)=-1.11, p=.276$.

Alternative moderators. We also calculated two regression analyses in which social orientation was replaced by either affect in general or anxiety in particular (assessed with PANAS), with Cyberball experience, substance, and all interaction terms as predictors (for details, see Supplemental Materials). We found no significant three-way interaction, $p$ s $>.579$. Thus, affect or anxiety did not qualify as moderators of oxytocin effects on social comfort. Also, affect and anxiety were not correlated with social orientation, $r \mathrm{~s}=-.04$ and .08 , respectively, $p \mathrm{~s}>.512$.

\section{Discussion}

In our study, responses to ostracism depended on the interplay between oxytocin, a neuropeptide with demonstrated prosocial effects (e.g., Kosfeld et al., 2005), and participants' social orientation. Oxytocin alleviated negative responses to an ostracism experience, specifically the drop in experienced social comfort, in those participants who were more (vs. less) motivated to sustain and promote social connectedness with others, as indicated by a greater orientation toward collectivism, especially of the horizontal type. This protective effect was not found for participants with a relatively greater orientation toward individualism. Hence, the administration of oxytocin did not indiscriminately induce 
participants to retain a sense of social comfort. Rather, the effect emerged only in combination with a more collectivistic (vs. individualistic) orientation. As such, our findings offer an explanation for why oxytocin did not shield against negative responses to ostracism in the study by Alvares et al. (2010): Oxytocin effects may have remained undetected because individual-differences in collectivistic (vs. individualistic) orientation were not taken into account.

According to our rationale, oxytocin can attenuate ostracism effects by inhibiting discomfort and withdrawal responses to adverse social experiences (Kemp \& Guastella, 2011; Radke et al., 2013). Note that this mechanism does not rely on increased salience or amplification of social cues. An amplification view would predict enhanced negative responses to social cues that indicate an antagonistic, or "unsafe," social environment (Olff et al., 2013). However, in our study responses to an uncomfortable social situation (ostracism) were not more negative in the oxytocin (vs. placebo) group (see Table 2).

Previous studies found that oxytocin has prosocial effects when the context is more (vs. less) conducive to prosocial interaction (Declerck, Boone, \& Kiyonari, 2010; De Dreu et al., 2010; De Dreu, Greer, Van Kleef, Shalvi, \& Handgraaf, 2011; for reviews, see Bartz et al., 2011; De Dreu, 2012a). Our results extend beyond this observed context-dependency of oxytocin effects by demonstrating that these effects can be qualified by individual differences, specifically, individuals' sense of being socially anchored. Apparently, oxytocin increase alone is not sufficient for socially protective effects; rather, a more (vs. less) collectivistic worldview allows these effects to unfold. Social orientation has been found to moderate oxytocin effects on other socially relevant emotional responses (Kim et al., 2011). Thus, we suspect that a collectivistic worldview not only shapes oxytocin effects in the context of ostracism, but might moderate oxytocin's impact in other social contexts as well.

Our findings are consistent with previous studies that have found a moderation of oxytocin effects on social cognition and behavior by people's relationship experiences and 
preexisting socio-emotional response tendencies (Bakermans-Kranenburg \& Van IJzendoorn, 2013; Olff et al., 2013). Specifically, the study by Radke et al. (2013) similarly revealed more social approach to adverse social stimuli in participants who felt generally safer in social situations and less socially anxious. However, two studies (De Dreu, 2012b; Luminet, Grynberg, Ruzette, \& Mikolajczak, 2011) have found a pattern that may seem opposite to the observed in our study and the study by Radke et al.: Oxytocin had more beneficial social effects in participants scoring lower on socio-emotional functioning (higher attachment avoidance or alexithymia). A closer inspection guided by our conceptualization suggests these studies differ from the present study and the study by Radke et al. in at least one critical aspect: The experimental tasks assessed prosocial cognition and behavior rather than responses to adverse social events (e.g., ostracism or antagonistic social stimuli). Thus, when it comes to prosocial responses in a safe interpersonal environment (as in De Dreu, 2012b, and Luminet et al., 2011), oxytocin may help people who tend to avoid social interaction to overcome these habitual tendencies. In contrast, when it comes to coping with antagonistic social experiences (as in our study and Radke et al.), oxytocin may be especially effective (i.e., protective) in people who feel more safely anchored in their close social relationships. Negative feelings from ostracism are part of an evolved alarm system that signals unsafe, potentially harmful social conditions (Gruter \& Masters, 1986). Could the observed attenuation of negative ostracism effects in collectivists, then, be maladaptive? We do not think so. Oxytocin would have maladaptive effects if it eliminated any negative response and thus forestalled the desire to re-affiliate. However, all of our participants, even those who had a more collectivistic worldview and were given oxytocin, reported lower social comfort after ostracism (vs. inclusion). Thus, there was clear evidence for sensitivity to the adverse social experience in those participants. The attenuation of pain from the single ostracism experience to a more acceptable level might allow people to engage more easily and quickly in re- 
affiliation attempts (Bernstein \& Claypool, 2012), which can help restore the threatened need states (Williams \& Nida, 2011). The present effect may be functional in this regard.

Could individual factors other than collectivistic orientation account for our findings? Collectivism might be (negatively) correlated with social anxiety, which has been found to moderate oxytocin effects on social approach (Radke et al., 2013), or rejection sensitivity, which involves a negative affective response to ostracism (Ayduk, Gyurak, \& Luerssen, 2008). However, in our study affect and the anxiety subscore were not correlated with collectivism and did not moderate oxytocin effects on responses to ostracism. Thus, there was no evidence that the observed pattern is merely a by-product of a more general, anxiolytic effect of oxytocin.

Self-reported childhood caregiving experience, which could be associated with horizontal collectivistic orientation, has also been found to moderate prosocial oxytocin effects (Bakermans-Kranenburg \& Van IJzendoorn, 2013; Riem, Bakermans-Kranenburg, Huffmeijer, \& van IJzendoorn, 2013). Whereas we did not examine the role of past caregiving experiences in the present study, we think that collectivism is a more critical and proximal construct because it captures people's current construal and experience of social life.

Our measure of social comfort consisted of two scales that are frequently employed in ostracism research, sense of belonging and socially reflected self-esteem. Consistent with our rationale, these two needs are often seen as closely related in the ostracism literature, that is, as components of a common "inclusionary" cluster (Williams, 2009). Both of these needs have been linked to re-affiliation behaviors (Williams, 2009), and have been shown to mediate affiliative responses to ostracism (Bernstein et al., 2010). The present effect of oxytocin, a neuropeptide often serving affiliative needs, is consistent with, and underscores, the proposed inclusionary component of the two constructs.

In conclusion, our study adds to the growing body of studies revealing the interplay between neuroendocrinological processes and individuals' construal of social life. The results 
of our study can be explained by a synergy of analogous effects exerted by oxytocin and collectivistic orientation. Oxytocin inhibits discomfort and withdrawal in antagonistic social situations and collectivism entails a firm sense of social connectedness. Oxytocin may act as an additional factor, a "catalyst," that permits this protective predisposition to become effective. It is possible that collectivists are more sensitive to exogenous oxytocin, such that the neuropeptide can more easily affect their responses to social exclusion. Further research is needed to pinpoint these processes in more detail. In any case, the present effects, if further corroborated, could also be harnessed in applied domains to help people cope with experiences of rejection, loneliness, or exclusion. 


\section{References}

Aiken, L. S., \& West, S. G. (1991). Multiple Regression: Testing and interpreting interactions. Thousand Oaks: Sage.

Alvares, G. A., Hickie, I. B., \& Guastella, A. J. (2010). Acute effects of intranasal Oxytocin on subjective and behavioral responses to social rejection. Experimental and Clinical Psychopharmacology, 18, 316-321.

Ayduk, Ö., Gyurak, A., \& Luerssen, A. (2008). Individual differences in the rejectionaggression link in the hot sauce paradigm: The case of rejection sensitivity. Journal of Experimental Social Psychology, 44, 774-782.

Bakermans-Kranenburg, M. J., \& Van Ijzendoorn, M. H. (2013). Sniffing around oxytocin: Review and meta-analyses of trials in healthy and clinical groups with implications for pharmacotherapy. Translational Psychiatry, 3, 1-14. doi:10.1038/tp.2013.34

Bartz, J. A., Zaki, J., Bolger, N., \& Ochsner, K. N. (2011). Social effects of oxytocin in humans: Context and person matters. Trends in Cognitive Sciences, 15, 301-309.

Bernstein, M. J., \& Claypool, H. M. (2012). Social exclusion and pain sensitivity: Why exclusion sometimes hurts and sometimes numbs. Personality and Social Psychology Bulletin, 38, 185-196.

Bernstein, M. J., Sacco, D. F., Brown, C. M., Young, S. G., \& Claypool, H. M. (2010). A preference for genuine smiles following social exclusion. Journal of Experimental Social Psychology, 46, 196-199.

Born, J., Lange, T., Kern, W., McGregor, G. P., Bickel, U., \& Fehm, H. L. (2002). Sniffing neuropeptides: A transnasal approach to the human brain. Nature Neuroscience, 5, 514516.

Dawson, J. F., \& Richter, A. W. (2006). Probing three-way interaction in moderated multiple regression: Development and application of a slope difference test. Journal of Applied Psychology, 91, 917-926. 
Declerck, C. H., Boone, C., \& Kiyonari, T. (2010). Oxytocin and cooperation under conditions of uncertainty: The modulating role of incentives and social information. Hormones and Behavior, 57, 368-374.

De Dreu, C. K. W. (2012a). Oxytocin modulates cooperation within and competition between groups: An integrative review and research agenda. Hormones and Behavior, 61, 419428.

De Dreu, C. K. W. (2012b). Oxytocin modulates the link between adult attachment and cooperation through reduced betrayal aversion. Psychoneuroendocrinology, 37, 871880.

De Dreu, C. K. W., Greer, L. L., Handgraaf, M. J. J., Shalvi, S., Van Kleef, G. A., Baas, M., Ten Velden, F. S., Van Dijk, E., \& Feith, S. W. W. (2010). The neuropeptide oxytocin regulates parochial altruism in intergroup conflict among humans. Science, 328, 14081411.

De Dreu, C. K. W., Greer, L. L., Van Kleef, G. A., Shalvi, S., \& Handgraaf, M. J. J. (2011). Oxytocin promotes human ethnocentrism. PNAS, 108, 1262-1266.

DeWall, C. N., MacDonald, G., Webster, G. D., Masten, C. L., Baumeister, R. F., Powell, C., Combs, D., Schurtz, D. R., Stillman, T. F., Tice, D. M., \& Eisenberger, N. I. (2010). Acetaminophen reduces social pain: Behavioral and neural evidence. Psychological Science, 21, 931-937.

Eisenberger, N. I., Lieberman, M. D., \& Williams, K. D. (2003). Does exclusion hurt? An fMRI study of social exclusion. Science, 302, 290-292.

Evans, S., Shergill, S. S., \& Averbeck, B. B. (2010). Oxytocin decreases aversion to angry faces in an associative learning task. Neuropsychopharmacology, 35, 2502-2509.

Graupmann, V., Jonas, E., Meier, E., Hawelka, S., \& Aichhorn, M. (2012). Reactance, the self, and its group: When threats to freedom come from the ingroup versus the outgroup. European Journal of Social Psychology, 42, 164-173. 
Grippo, A. J., Trahanas, D. M., Zimmerman, R. R., Porges, S. W., \& Carter, C. S. (2009). Oxytocin protects against negative behavioral and autonomic consequences of long-term social isolation. Psychoneuroendocrinology, 34, 1542-1553.

Gruter, M., \& Masters, R. D. (1986). Ostracism: A social and biological phenomenon. Ethnology and Sociobiology, 7, 149-395.

Huang, J. Y., Ackerman, J. M., \& Bargh, J. A. (2013). Superman to the rescue: Simulating physical invulnerability attenuates exclusion-related interpersonal biases. Journal of Experimental Social Psychology, 49, 349-354.

Kemp, A. H., \& Guastella, A. J. (2011). The role of oxytocin in human affect: A novel hypothesis. Current Directions in Psychological Science, 20, 222-231.

Kim, U. (1994). Individualism and collectivism: Conceptual clarification and elaboration. In U. Kim, H. C. Triandis, C. Kagitçibasi, S-C. Choi, \& G. Yoon (Eds.), Individualism and collectivism. Theory, method, and applications (pp. 19-40). Thousand Oaks, CA: Sage.

Kim, H. S., Sherman, D. K., Mojaverian, T., Sasaki, J. Y., Park, J., Suh, E. M., \& Taylor, S. E. (2011). Gene-culture interaction: Oxytocin receptor polymorphism (OXTR) and emotion regulation. Social Psychological and Personality Science, 2, 665-672.

Knowles, M. L. (2013). Belonging regulation through the use of (para)social surrogates. In N. DeWall (Ed.), The Oxford handbook of social exclusion (pp. 275-284). Oxford: Oxford University Press.

Kosfeld, M., Heinrichs, M., Zak, P. J., Fischbacher, U., \& Fehr, E. (2005). Oxytocin increases trust in humans. Nature, 435, 673-676.

Luminet, O., Grynberg, D., Ruzette, N., \& Mikolajczak, M. (2011). Personality-dependent effects of oxytocin: Greater social benefits for high alexithymia scorers. Biological Psychology, 87, 401-406.

Markus, H. R., \& Kitayama, S. (1991). Culture and the self: Implications for cognition, emotion, and motivation. Psychological Review, 98, 224-253. 
Olff, M., Frijling, J. L., Kubzansky, L. D., Bradley, B., Ellenbogen, M. A., Cardoso, C., Bartz, J. A., Yee, J. R., \& van Zuiden, M. (2013). The role of oxytocin in social bonding, stress regulation and mental health: An update on the moderating effects of context and interindividual differences. Psychoneuroendocrinology, 38, 1883-1894.

Oyserman, D., Coon, H. M., \& Kemmelmeier, M. (2002). Rethinking individualism and collectivism: Evaluation of theoretical assumptions and meta-analyses. Psychological Bulletin, 128, 3-72.

Oyserman, D. \& Lee, S. (2008). Does culture influence what and how we think? Effects of priming individualism and collectivism. Psychological Bulletin 134, 311-342.

Pfundmair, M., Graupmann, V., Frey, D., \& Aydin, N. (2014). The different behavioral intentions of collectivists and individualists in response to social exclusion. Manuscript submitted for publication.

Radke, S., Roelofs, K., \& De Bruijn, E. R. A. (2013). Acting on anger: Social anxiety modulates approach-avoidance tendencies after oxytocin administration. Psychological Science, 24, 1573-1578.

Ren, D., Wesselmann, E. D., \& Williams, K. D. (2013). Interdependent self-construal moderates coping with (but not the initial pain of) ostracism. Asian Journal of Social Psychology. doi:10.1111/ajsp.12037

Riem, M. M. E., Bakermans-Kranenburg, M. J., Huffmeijer, R., \& Van IJzendoorn, M. H. (2013). Does intranasal oxytocin promote prosocial behavior to an excluded fellow player? A randomized-controlled trial with Cyberball. Psychoneuroendocrinology, 38, 1418-1425.

Singelis, T. M., Triandis, H. C., Bhawuk, D., \& Gelfand, M. (1995). Horizontal and vertical individualism and collectivism: A theoretical and methodological refinement. Journal of Cross-Cultural Research, 29, 240-275.

Triandis, H. C. (1995). Individualism and collectivism. Boulder, CO: Westview Press. 
Triandis, H. C., \& Gelfand, M. J. (1998). Converging measurement of horizontal and vertical individualism and collectivism. Journal of Personality and Social Psychology, 74, 118128.

Triandis, H. C., \& Gelfand, M. J. (2012). A theory of individualism and collectivism. In P. A. M. Van Lange, A. W. Kruglanski \& E. T. Higgins (Eds.), Handbook of theories of social psychology (Vol. 2, pp. 498-520). Los Angeles: Sage.

Watson, D., Clark, L. A., \& Tellegen, A. (1988). Development and validation of brief measures of positive and negative affect: The PANAS scales. Journal of Personality and Social Psychology, 54, 1063-1070.

Wesselmann, E. D., Wirth, J. H., Pryor, J. B., Reeder, G. D., \& Williams, K. D. (2013). When do we ostracize? Social Psychological and Personality Science, 4, 108-115.

Williams, K. D. (2007). Exclusion. Annual Reviews of Psychology, 58, 425-453.

Williams, K. D. (2009). Ostracism: Effects of being excluded and ignored. In M. P. Zanna (Ed.), Advances in experimental social psychology (Vol. 41, pp. 275-314). New York: Academic press.

Williams, K. D., Cheung, C. K. T., \& Choi, W. (2000). Cyberostracism: Effects of being ignored over the Internet. Journal of Personality and Social Psychology, 79, 748-762.

Williams, K. D., \& Jarvis, B. (2006). Cyberball: A program for use in research on interpersonal ostracism and acceptance. Behavior Research Methods, 38, 174-180.

Williams, K. D., \& Nida, S. A. (2011). Ostracism: Consequences and coping. Current Directions in Psychological Science, 20, 71-75.

Zadro, L., Williams, K. D., \& Richardson, R. (2004). How low can you go? Exclusion by a computer is sufficient to lower self-reported levels of belonging, control, self-esteem, and meaningful existence. Journal of Experimental Social Psychology, 40, 560-567. 


\section{Footnotes}

${ }^{1}$ While the terms ostracism, exclusion, and rejection have been used interchangeably in earlier research, we would like to draw attention to critical differences. Ostracism is defined as being ignored and excluded, and it often occurs without explanation or explicit negative attention, typically during an unfolding sequence of behaviors. Social exclusion is defined as being excluded, alone, or isolated with or without explicit declarations of dislike, and occurs after initial interaction and subsequent separation from others or as a hypothetical consequence in the future. Rejection involves an explicit declaration by an individual or group that they do not want to interact with a target individual, and also occurs after interaction and separation (Williams, 2007). The distinctiveness of the three terms is supported by empirical research, which has shown that manipulations designed to affect one of the three constructs lead to different consequences. In our research, we use a well-established manipulation of ostracism, so that we stick to this term consistently.

${ }^{2}$ Social orientation was measured immediately after substance administration. The completion of the measure (IND-COL) took approximately $5 \mathrm{~min}$, and intranasally administered oxytocin is known to become effective after a delay of approximately $40 \mathrm{~min}$. Thus, the substance should not affect social orientation responses. Indeed, there was no evidence that participants in the oxytocin and placebo groups differed in this respect (see Manipulation Check).

${ }^{3}$ The measures employed by Zadro et al. (2004) also cover sense of control and meaningful existence. We focused on responses relevant to the social effects of oxytocin. However, the pattern of findings remained essentially unchanged when control/ meaningful existence were included in the outcome score (see Supplemental Material). 
Table 1

Results for the multiple regression with Cyberball experience, substance, social orientation, and all interaction terms as predictors of the main dependent measure (social comfort in response to the Cyberball game)

\begin{tabular}{|c|c|c|c|c|c|}
\hline & $\begin{array}{l}\text { Unstandardized } \\
\text { regression } \\
\text { coefficients }(b)\end{array}$ & $\begin{array}{c}\text { Standard error } \\
\text { for } b \text { estimates } \\
(S E)\end{array}$ & $\begin{array}{c}\text { Standardized } \\
\text { regression } \\
\text { coefficients }(\beta)\end{array}$ & $t$ & $p$ \\
\hline Cyberball experience & -1.170 & .15 & -.68 & -8.00 & $<.001 * *$ \\
\hline Substance & 0.115 & .15 & .07 & 0.78 & .436 \\
\hline Social orientation & 0.204 & .15 & .12 & 1.35 & .181 \\
\hline Cyberball experience X Substance & 0.094 & .15 & .06 & 0.64 & .522 \\
\hline Cyberball experience X Social orientation & 0.095 & .15 & .06 & 0.63 & .530 \\
\hline Substance X Social orientation & 0.120 & .15 & .07 & 0.79 & .431 \\
\hline $\begin{array}{l}\text { Cyberball experience X Substance X Social } \\
\text { orientation }\end{array}$ & 0.388 & .15 & .22 & 2.57 & $.012 *$ \\
\hline$R^{2}$ & .510 & & & & \\
\hline Adjusted $R^{2}$ & .461 & & & & \\
\hline
\end{tabular}

$* * p<.001, * p<.05$ 
Table 2

Means and standard deviations (in parenthesis) of social comfort as a function of Cyberball experience (ostracism vs. inclusion) and substance (oxytocin vs. placebo)

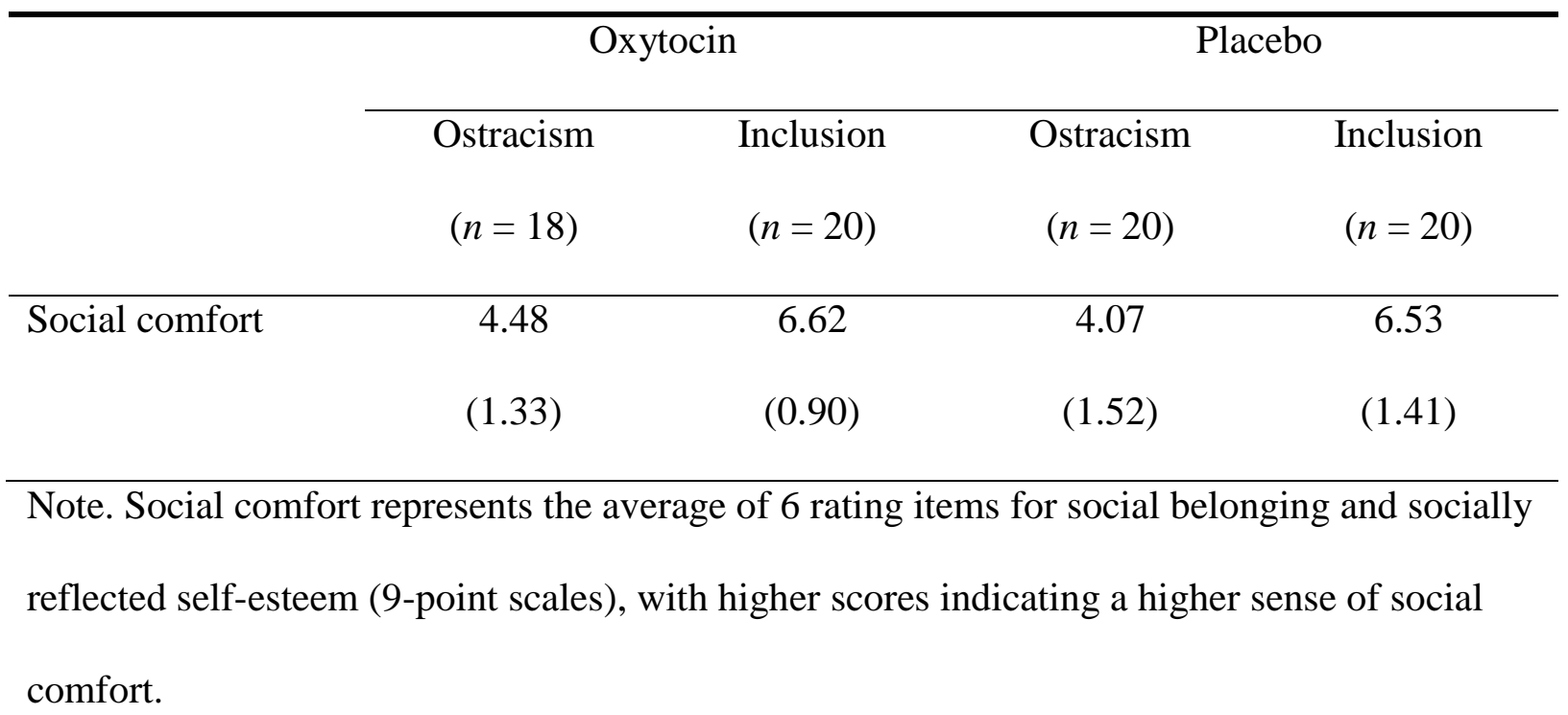



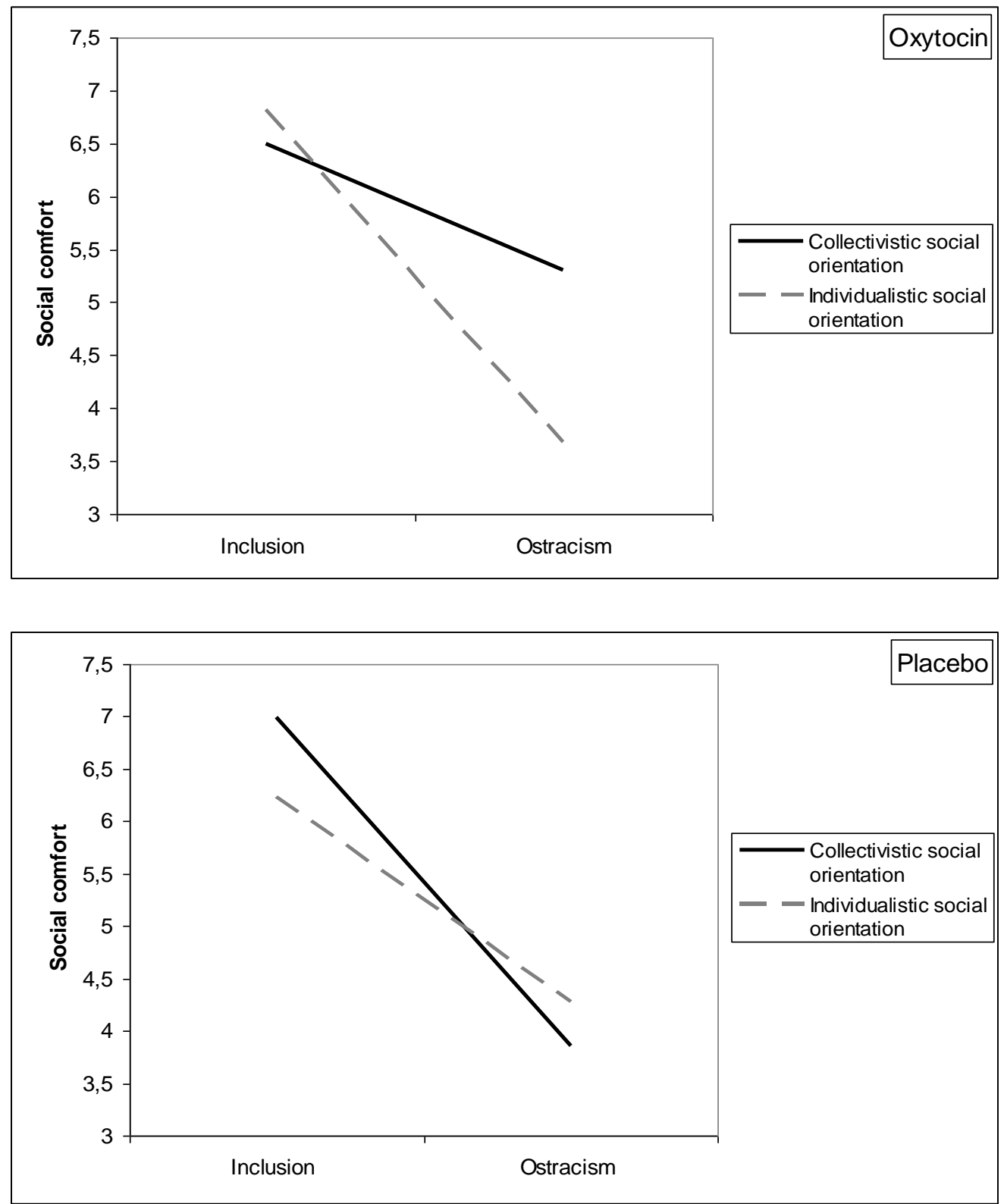

Figure 1. Social comfort in response to the Cyberball game as a function of Cyberball experience (ostracism vs. inclusion) and social orientation (plotted at $1 S D$ above vs. below the mean) for the oxytocin (top) and placebo group (bottom) 